\title{
Parafusos pediculares e violação facetária - A importância do ângulo entre a faceta e o parafuso
}

\section{Pedicle Screws and Facet Violation - The importance of the Angle between the Facet and the Screw}

\author{
Rodrigo Amaral ${ }^{1}$ Luiz Pimenta ${ }^{1,2(0)}$ Angelo Guarçoni Netto ${ }^{1(0)}$ Gabriel Henrique Pokorny ${ }^{1(0)}$ \\ Raquel Fernandes 1 (1) \\ ${ }^{1}$ Instituto de Patologia da Coluna (IPC), São Paulo, SP, Brasil \\ 2 University of California, UCSD, San Diego, CA, Estados Unidos da América \\ Endereço para correspondência Gabriel Henrique Pokorny, B Sc., \\ Rua Vergueiro, 1421, São Paulo, SP, Brasil (e-mail: ghpokorny@gmail.com).
}

Rev Bras Ortop 2020;55(5):642-648.

\section{Resumo \\ Palavras-chave \\ - parafusos pediculares \\ - fusão vertebral \\ - tomografia computadorizada por raios $\mathrm{x}$ \\ - estudos retrospectivos}

\section{Abstract}

Objetivo Verificar se, independente da técnica de colocação do parafuso, há uma distância ou angulação segura em relação as facetas para que os parafusos sejam colocados de modo a evitar a violação da articulação facetária.

Métodos Estudo retrospectivo, comparativo, não randomizado, em centro único. Foram avaliados em tomografia computadorizada axial: o ângulo do parafuso/barra em relação a linha média, o ângulo do centro das facetas em relação a linha média, a distância entre a cabeça do parafuso/barra até a linha média, e a distância do centro das facetas até a linha média; a violação da articulação facetária será avaliada em uma gradação de 0 a 2 . Serão também calculados a diferença entre o ângulo do parafuso e ângulo da faceta ( $\triangle$ Ångulo) e também a diferença entre a distância da faceta e a distância do parafuso ( $\Delta$ Distância).

Resultados Um total de 212 pacientes e 397 facetas foram analisados (196 do lado esquerdo e 201 do lado direito). Destes, 303 foram não violados (grau 0), correspondendo a 76,32\%, e 94 sofreram algum tipo de violação (grau 1 e 2), correspondendo a $23,68 \%$. A média do $\Delta$ ângulo foi de $9,87^{\circ}+/-4,66^{\circ}$ (grau 0 ) e de $3,77^{\circ}+/-4,93^{\circ}$ em facetas (grau 1 e 2) $(p<0.001)$, e o $\Delta$ distância médio nos casos em que não houve violação foi de 0,94 unidades aleatórias (u.a.) $+/-0,39$ u.a., enquanto o $\Delta$ distância de casos G1 e G2 foi de 0,56 u.a. + | 0,25 u.a. $(p<0.001)$.

Conclusão As medidas de ângulo e distância entre faceta e parafuso, podem auxiliar na colocação de parafusos. Esses parâmetros podem ser utilizados como medidas de segurança com o uso mais frequentes das técnicas de navegação cirúrgica.

Objective To verify whether, regardless of the screw placement technique, there is a safe distance or angle in relation to the facets that can prevent violation of the facet joint when the screws are placed.

Methods Retrospective, single, comparative, non-randomized center. We evaluated by axial computed tomography: the angle of the screw/rod in relation to the midline, the angle recebido

18 de Setembro de 2019

aceito

27 de Janeiro de 2020
DOI https://doi.org/

10.1055/s-0040-1709200. ISSN 0102-3616.
Copyright $\odot 2020$ by Sociedade Brasileira License terms de Ortopedia e Traumatologia. Published by Thieme Revinter Publicações Ltda, Rio de Janeiro, Brazil 
Keywords

- pedicle screws

- spinal fusion

- x-ray computed tomography

- retrospective studies of the center of the facets in relation to the midline, the distance between the head of the screw/rod to the midline, and the distance from the center of the facets to the midline; the violation of the facet joint will be evaluated in a gradation of 0 to 2 . Also will be measured the difference between the angle os the facets and the angle of the screws ( $\Delta$ Angle) and, the difference between the facet distance and the screw distance ( $\Delta$ Distance).

Results A total of 212 patients and 397 facets were analyzed (196 on the left and 201 on the right). Of these, 303 were not violated (grade 0 ), corresponding to $76,32 \%$, and 94 suffered some type of violation (grade 1 and 2 ), corresponding to $23,68 \%$. The mean of $\Delta$ angle was $9.87^{\circ}+1-4.66^{\circ}$ (grade 0 ), and of $3.77^{\circ}+1-4.93^{\circ}$ in facets (grade 1 and 2) $(p<0.001)$, and the $\Delta$ mean distance in cases in which there was no violation was 0.94 arbitrary units (a.u.) $+1-0.39$ a.u., while the $\Delta$ distance in $\mathrm{G} 1$ and $\mathrm{G} 2$ cases was 0.56 a.u. $+\mid-0.25$ a.u. $(p<0.001)$.

Conclusion The measurements of angle and distance between facet and screw can help in the placement of screws. These parameters can be used as safety measures with the most frequent use of surgical navigation techniques.

\section{Introdução}

Problemas relacionados à coluna lombar estão se tornando cada vez mais frequentes com o aumento da expectativa de vida e consequente envelhecimento da população. ${ }^{1}$ Por provocarem a redução de mobilidade, dores mecânicas, e redução na qualidade de vida, estes se tornaram uma das principais causas de incapacitação da população mundial. ${ }^{2}$ Por vezes, o tratamento dessas dores pode se dar de maneira conservadora; no entanto, muitos pacientes se apresentam, por vezes, refratários a estes tratamentos, e a maioria destes requer o tratamento cirúrgico para resolução dos seus sintomas. ${ }^{3}$

A depender do tipo de condição a ser tratada, faz-se necessário o uso de suplementação/fixação posterior. ${ }^{4,5}$ Múltiplas técnicas para suplementação/fixação lombar foram propostas e utilizadas, entretanto por reduzirem o risco de lesões neurovasculares e por promoverem grande estabilização da construção, parafusos pediculares se tornaram o método de preferência para a fixação posterior em cirurgias lombares permitindo a estabilidade do nível operado. $^{6-8}$

Diversas técnicas são propostas para a colocação de parafusos pediculares, entre elas as técnicas de Magerl, ${ }^{9}$ Roy-Camille et al., ${ }^{10}$ e Weinsntein et al., ${ }^{11}$ dentre outras. ${ }^{12}$ Além disso, com a popularização de técnicas minimamente invasivas para cirurgias da coluna, vem aumentando em quantidade a colocação de parafusos pediculares por técnica percutânea. ${ }^{13,14}$

Contudo, um problema que muitas vezes não é levado em consideração em relação a colocação destes parafusos é a violação da capsula facetária. ${ }^{6,8,14}$ Estudos recentes demonstram taxas que variam entre 0 e $80 \%$ de violação facetaria do nível adjacente à vértebra instrumentada mais cranial, que variam de acordo com a técnica utilizada e experiência do cirurgião. ${ }^{15,16}$

Essa violação facetária do nível cranial mais instrumentado é um dos fatores que é apontado por estudos como fator de risco para o desenvolvimento de doença do nível adjacente que pode levar à degeneração do mesmo, podendo acarretar em piora da qualidade de vida e futura necessidade de reoperação para o tratamento deste nível. ${ }^{8,17}$

Deste modo, o objetivo do nosso trabalho é verificar se, independente da técnica de colocação do parafuso, há uma distância ou angulação segura em relação às facetas, para que os parafusos sejam colocados de modo a evitar a violação da articulação facetária.

\section{Materiais e métodos}

O presente estudo é retrospectivo, comparativo, não randomizado, realizado em centro único, e foi aprovado pelo conselho de ética em pesquisa (CEP) da instituição sob o N ○ 91876318.0.0000.8098.

Foram incluídos no estudo pacientes que possuíam fixação por parafusos pediculares na coluna lombar e que possuíam exames de tomografia computadorizada com cortes axiais de janela óssea. Pacientes cujos exames de tomografia computadorizada estavam com qualidade ruim não permitindo boa visualização das estruturas necessárias para o estudo foram excluídos, assim como casos envolvendo fraturas, malformações ou outras condições que não permitiriam a boa visualização das estruturas necessárias para o estudo.

A avaliação foi realizada por dois pesquisadores diferentes, sendo um terceiro avaliador chamado para resolver qualquer discordância entre as medidas. Foram medidos, nas imagens radiológicas de tomografia computadorizada em corte axial em janela óssea, os seguintes parâmetros: ângulo do parafuso/barra em relação a linha média, ângulo do centro das facetas em relação a linha média e a diferença entre o ângulo de parafuso e ângulo da faceta ( $\Delta$ ângulo) ( - Figura 1 A-C), distância entre a cabeça do parafuso/barra até a linha média, distância do centro das facetas até a linha média* ( ${ }^{*}$ as distâncias serão medidas em unidades 


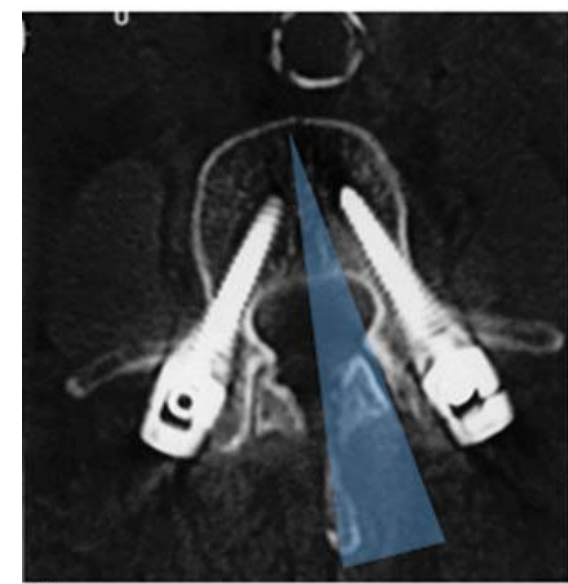

A

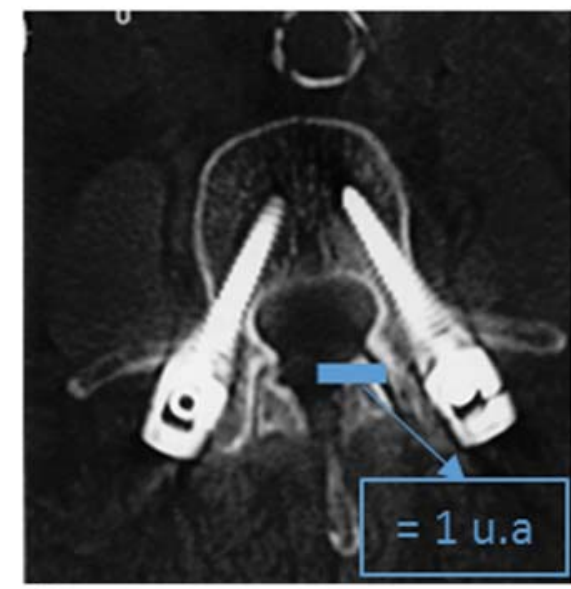

D

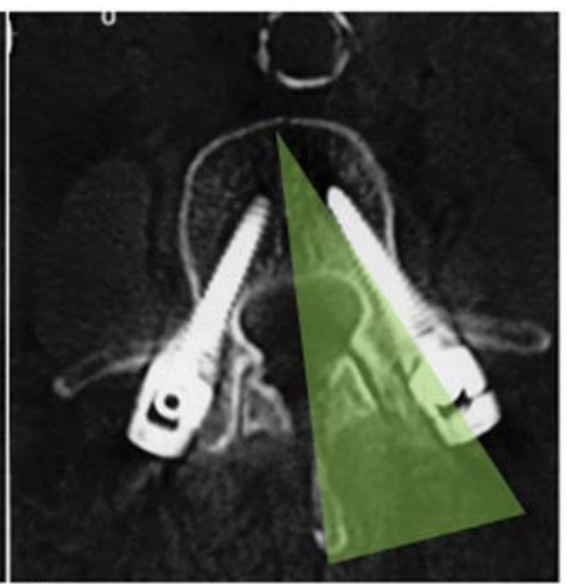

B

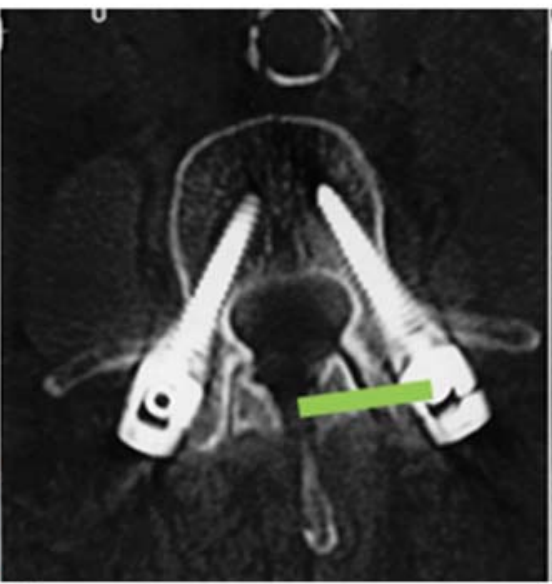

E

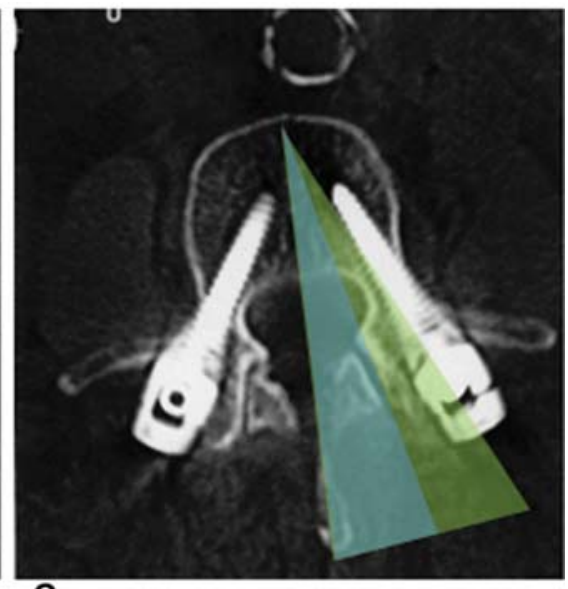

C

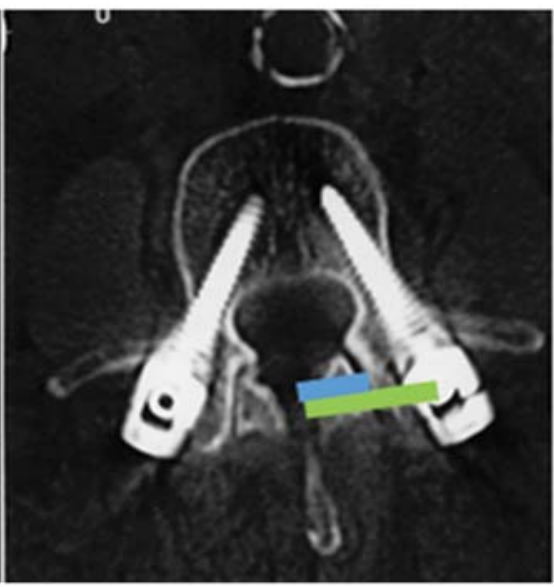

$\mathbf{F}$

Fig. 1 Figura representando as medidas realizadas durante o trabalho. (A) Angulação das facetas; (B) Angulação do parafuso/barra; (C) $\Delta$ facetas; (D) distância da faceta; (E) Distância parafuso/barra; (F) $\Delta$ distância. (Azul: medidas da faceta; Verde: medidas dos parafusos/barras).

arbitrárias (u.a.), sendo a distância entre o centro das facetas e a linha média da vertebra igual a 1 u.a.), e a diferença entre o valor de distância do parafuso e distância da faceta $(\Delta$ distância) (—Figura 1 D-F).

A violação da articulação facetária foi avaliada em uma gradação de 0 a 2 de acordo com a classificação proposta por Tannous et al. ${ }^{18}$

Os dados foram compilados no programa Excel (Microsoft Corp., Redmond, WA, EUA) e analisados estatisticamente através dos programas GraphPad Prism 8 (GraphPad Software Inc, La Jolla, CA, EUA). Para testar diferenças entre medidas quantitativas paramétricas, foi utilizado o teste-t de Student, e para valores fora da distribuição normal foi utilizado o teste de Mann-Witney. Para a comparação de medidas qualitativas, foi utilizado o teste de Fischer ou o de Qui-quadrado. Resultados com valores de $p$ menor do que 0,05 foram considerados como estatisticamente significantes.

\section{Resultados}

\section{Demográficos}

Um total de 212 pacientes foram incluídos no estudo, sendo um total de 397 facetas analisadas (196 do lado esquerdo e 201 do lado direito). Não houve diferença no número de

Tabela 1 Tabela demonstrando o número de violações identificadas durante o estudo

\begin{tabular}{|l|l|l|l|}
\hline & Esquerda & Direita & Total \\
\hline Total & 196 & 201 & 397 \\
\hline Violação grau 0 (\%) & $151(77 \%)$ & $152(76 \%)$ & $303(76 \%)$ \\
\hline Violação grau 1 (\%) & $22(11 \%)$ & $30(15 \%)$ & $52(13 \%)$ \\
\hline Violação grau 2 (\%) & $23(12 \%)$ & $19(9 \%)$ & $42(11 \%)$ \\
\hline
\end{tabular}


Tabela 2 Tabela demonstrando o número de violações identificadas por nível durante o estudo

\begin{tabular}{|l|l|l|l|}
\hline & Violação G0 & $\begin{array}{l}\text { Violação } \\
\text { G1 e G2 }\end{array}$ & \% de violação \\
\hline Torácica & 11 & 7 & 39,9 \\
\hline L1 & 11 & 10 & 47,6 \\
\hline L2 & 30 & 5 & 14,3 \\
\hline L3 & 50 & 20 & 28,6 \\
\hline L4 & 131 & 31 & 19,1 \\
\hline L5 & 70 & 21 & 23,1 \\
\hline
\end{tabular}

violações entre o lado esquerdo e o lado direito. Destas, 303 (77\%) facetas não sofreram violação (grau 0). Em 52 (13\%) casos, o parafuso encostou levemente na faceta (grau 1), e, em $42(10 \%)$ casos, houve violação da faceta (grau 2) $(p>0.05)$ (-Tabela 1$)$.

Foi visto também que os níveis T1-L1 foram os níveis mais suscetíveis à violação facetária $(p<0.05)$ (-Tabela 2 e - Figura 2).

\section{Angulação do parafuso e angulação das facetas}

A angulação média das facetas em relação à linha média foi de $18,9^{\circ}+1-3,95^{\circ}$, a angulação média dos parafusos em relação à linha média foi de $27,5^{\circ}+/-5,7^{\circ}$, e o $\Delta$ facetas médio foi de $+8,5^{\circ}$. A média do $\Delta$ ângulo foi de $9,87^{\circ}+1-$ $4,66^{\circ}$ (grau 0 ), e de $3,77^{\circ}+1-4,93^{\circ}$ em facetas onde houve violação (grau 1 e 2) $(p<0.001)$ (-Tabela 3 ).

Para fins de melhor análise e estratificação, foram definidos 3 pontos para a análise: $\Delta$ menor do que $5^{\circ}, \Delta$ ângulo entre $5^{\circ}$ e $15^{\circ}$, e $\Delta$ ângulo maior do que $15^{\circ}$. Em casos de $\Delta$ faceta menor do que $5^{\circ}$, houve violação das facetas em $65 \%$ (63/96) dos casos, ao passo que com $\Delta$ entre 5 e $15^{\circ}$ houve violação das facetas em 11\% (30/265) dos casos, e nos casos com angulação maior do que $15^{\circ}$, houve violação em apenas $2,7 \%$ dos casos (1/36) (-Figura 3 ).

\section{Distância do parafuso e distância das facetas}

A distância média entre os parafusos e a linha média foi de 1,85 u.a + / - 0,40 u.a, logo o $\Delta$ distância foi de 0,85 u.a + /0,40 u.a. Sendo que o $\Delta$ distância médio em casos onde não houve violação foi de 0,94 u.a $+\mid-0,39$ u.a, enquanto o $\Delta$ distância em casos de violação (g1 e g2) foi de 0,56 u.a +/0,25 u.a $(p<0.001)$ ( Tabela 4).

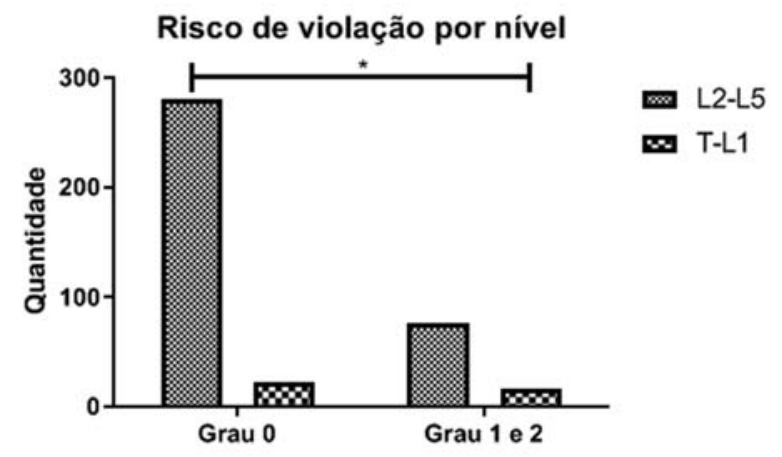

Fig. 2 Gráfico representando quantidade de violação entre os níveis T-L1 e L2-L5. ${ }^{*} p<0.05$.

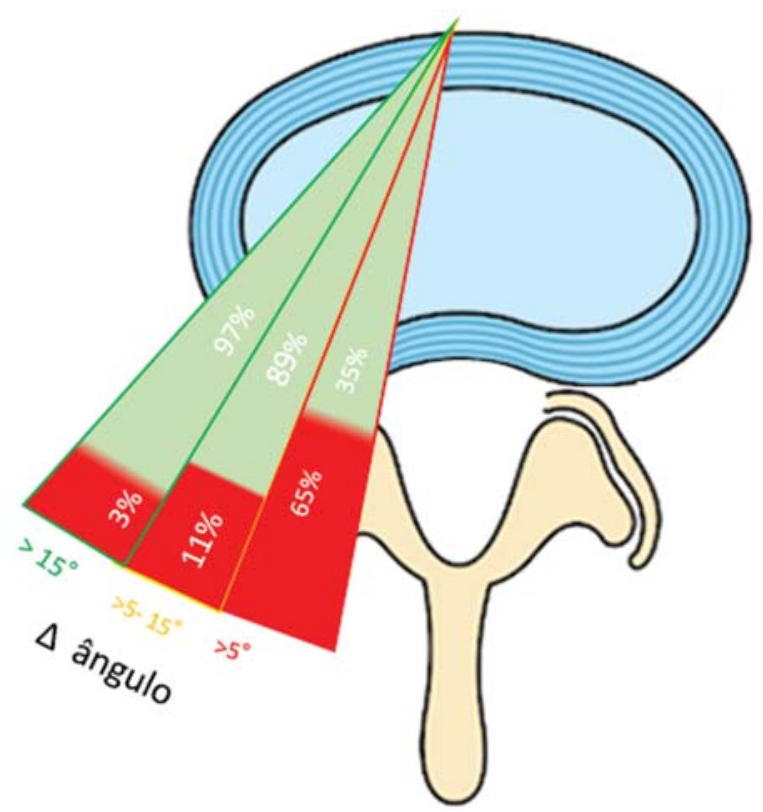

Fig.3 Imagem demonstrando a proporção de violação em relação ao $\Delta$ ângulo. Verde: representa não violação. Vermelha: representa violação.

Foram definidos por 3 pontos para melhor análise dos dados: 0,5 u.a; entre 0,5 e 1,5 u.a, e maior que 1,5 u.a. Sendo evidenciado que em casos de parafusos colocados a uma distância menor do que 0,5 u.a do centro das facetas houve

Tabela 3 Tabela demonstrando a média da angulação das facetas, angulação dos parafusos e $\Delta$ ângulo

\begin{tabular}{|l|l|l|l|}
\hline & $\begin{array}{l}\text { Angulação } \\
\text { facetas }\end{array}$ & $\begin{array}{l}\text { Angulação } \\
\text { parafusos }\end{array}$ & $\Delta$ Ângulo \\
\hline Média (DP) & $18^{\circ}+/-3,95^{\circ}$ & $27,5^{\circ}+1-5,7^{\circ}$ & $8,5^{\circ}+1-5,28^{\circ}$ \\
\hline$\Delta$ ângulo e violação & Grau 0 & Graus 1 e 2 & $P$ \\
\hline \multicolumn{2}{|l|}{} & $3,77^{\circ}+1-4,93^{\circ}$ & $<0,001$ \\
\hline Média (DP) & $9,87^{\circ}+/-4,66^{\circ}$ & \\
\hline
\end{tabular}

Abreviatura: DP, desvio padrão. 
Tabela 4 Tabela demonstrando a média da distância do parafuso e $\Delta$ distância

\begin{tabular}{|l|l|l|l|}
\hline & Distância parafuso & $\Delta$ distância & \\
\hline Média (+/- DP) & $1,85+/-0,40$ u.a & $0,85+/-0,40$ u.a & \\
\hline$\Delta$ ângulo e violação & Grau 0 & Graus 1 e 2 & $P$ \\
\hline \multicolumn{2}{|l|}{$0,56+/-0,25$ u.a } & $<0,001$ \\
\hline Média (+/- DP) & $0,94+/-0,39$ u.a & 0,56 & \\
\hline
\end{tabular}

Abreviaturas: DP, desvio padrão; u.a., unidades arbitrárias.

violação em 65\% dos casos (38/59), já em parafusos colocados em uma distância entre 0,5 e 1,5 u.a, houve violação em $17 \%$ dos casos (56/319), e, em parafusos colocados com mais de 1,5 u.a, não houve violação facetária $(0 / 19)(p<0.001)$ (-Figura 4).

curva característica de operação do receptor Para verificar a capacidade das medidas em indicarem quando houve ou não violação facetaria foi utilizado o método de curvas COR. As medidas angulação das facetas, $\Delta$ ângulo e $\Delta$ distância apresentaram as seguintes áreas sob a curva (AUC): 0.5848; 0.8599; 0.8195 (-Figura 5).

\section{Discussão}

Apesar de serem considerados o padrão-ouro de fixação posterior para coluna lombar, os parafusos pediculares

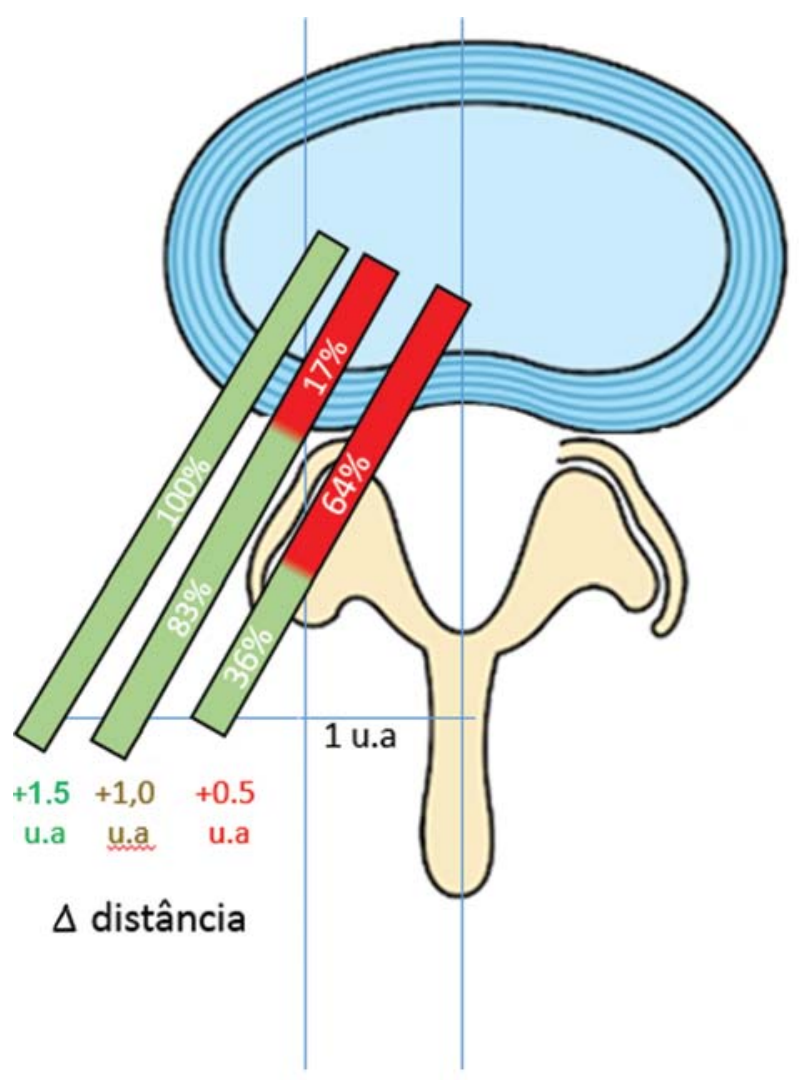

Fig.4 Imagem demonstrando a proporção de violação em relação ao $\Delta$ distância. Verde: representa não violação. Vermelha: representa violação. podem causar a violação das cápsulas facetárias caso não sejam colocados corretamente, fator que pode iniciar ou acelerar o processo de degeneração do nível adjacente., ${ }^{7,19}$

\section{Violação facetária versus técnica de parafusos pedicular}

Em um dos primeiros estudos a comparar o risco de violação facetária entre técnicas percutâneas e abertas, Babu et al. ${ }^{20}$ demonstraram que o uso de parafusos percutâneos trazia um maior risco de violação facetária do que a utilização de técnicas abertas ( $8,5 \%$ versus $2 \%, p<0.05$; RP 8.55). Esses resultados são similares aos encontrados por Jones-Quaidoo et al., ${ }^{13}$ que reportaram uma taxa de $13 \%$ de violação facetária com técnica percutânea e de $6 \%$ com o uso de técnica aberta. Dando um passo à frente, pesquisadores realizaram uma meta-análise comparando a taxa de violação facetária entre o uso de cirurgia robótica e colocação manual dos parafusos, demonstrando que o auxílio do robô reduziu o número de violações facetárias por parafusos pediculares. $^{21}$

Alguns pesquisadores também buscaram verificar o risco de violação da cápsula facetária entre os diversos tipos de inserção de parafusos pediculares. Matusakawa et al. ${ }^{8}$ reportaram uma taxa de $11 \%$ de violação facetária com o uso da técnica de trajetória de osso cortical (cortical bone trajectory". Outro estudo comparou a taxa de violação facetária entre a técnica lateral-to-medial (LMT, na sigla em inglês) e a técnica owl's eye (OET, na sigla em inglês), demonstrando que pacientes submetidos à OET possuíram maior quantidade de facetas superiores violadas. ${ }^{18}$

\section{Marcos anatômicos e violação facetária}

Apesar da identificação de técnicas que podem trazer mais riscos de violação, outro ponto importante é identificar estruturas e parâmetros que possam servir de referência para a localização e, teoricamente, para a redução de violação das facetas. ${ }^{6,17,22}$ Dentre os fatores apontados como tendo relação com a violação facetaria, Jia et al. ${ }^{23}$ evidenciaram que quanto mais inferior o nível, maior a taxa de violação facetária, similar aos achados de Teles et al., ${ }^{6}$ que reportaram $100 \%$ das violações facetárias nos níveis L4 e L5. Esses resultados são diferentes aos apontados pelo nosso estudo, que identificou os níveis torácicos e L1 como os mais suscetíveis à violação facetária.

Especula-se também na literatura se a angulação da faceta poderia influenciar no risco de violação facetária. Dois estudos recentes avaliaram a relação da angulação da faceta com a violação facetária; Teles et al. ${ }^{6}$ demonstraram que 


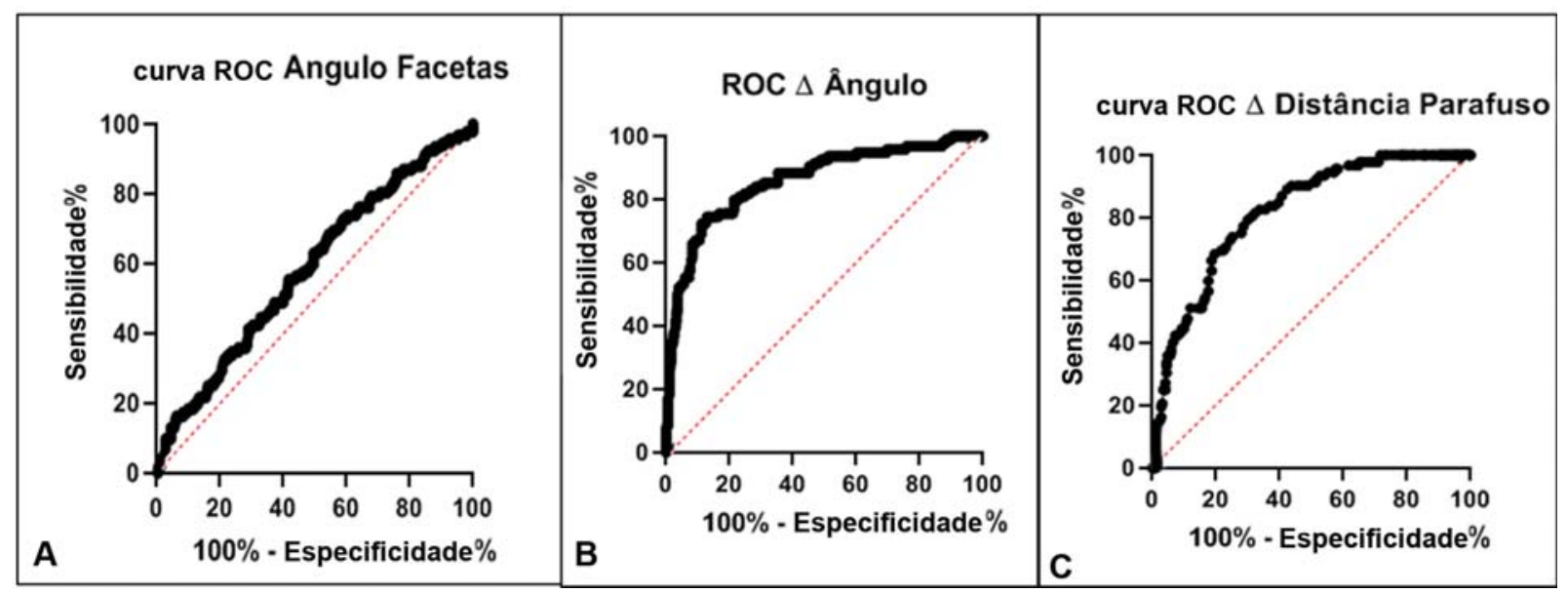

Fig. 5 Imagens demonstrando a curva característica de Receiver-Operating Curve (ROC) das diversas medidas demonstradas no trabalho. (A) Ângulo das facetas; (B) $\Delta$ ângulo e (C) $\Delta$ distância.

quanto maior a angulação da faceta, maior o risco de violação $\left(49^{\circ}\right.$ versus $\left.34^{\circ} ; p<0,001\right)$, do mesmo modo que o reportado por Xu et al. ${ }^{17}$ que relatou uma média de $39^{\circ}$ em casos onde houve violação e $33^{\circ}$ em casos sem violação $(p<0,001)$. Esses resultados são similares aos apresentados pelo nosso trabalho, onde o grupo sem violação teve em média $18^{\circ}$ contra $19^{\circ}$ no grupo onde houve violação $(p<0,05)$. No entanto, nós também comparamos a relação do ângulo da faceta com o ângulo do parafuso ( $\Delta$ ângulo) e identificamos que quanto menor a proporção entre os dois, maior era o risco de violação da cápsula facetária (g0 $9,8^{\circ}$ vs g1/2 3,7 ; $p<0,0001$ ). Ademais, nosso estudo demonstrou que a diferença de angulação entre o parafuso e o centro das facetas ( $\Delta$ ângulo) poderia indicar com mais precisão o risco de violação ou não da cápsula facetária, como demonstrado na - Figura $\mathbf{5}$, que compara a curva COR obtida tanto pelo uso do ângulo da faceta com o uso da medida $\Delta$ ângulo $(A U C=0,5848$ versus $\mathrm{AUC}=0,8599, p<0,001$ ).

A distância a qual o parafuso pedicular foi colocado das facetas também foi analisada pelo nosso estudo, onde demonstramos que quanto maior a distância do parafuso até o centro das facetas, menor o risco de violação facetária (g0: 0,94 u.a versus g1/2: 0,56 u.a; $p<0,001$ ). Esse resultado é similar ao obtido em um estudo que analisou o risco de violação facetária após a colocação de parafusos pediculares na trajetória do osso cortical; no entanto, nesse estudo, a distância do parafuso para a faceta superior foi medida verticalmente. $^{8}$

O presente estudo conta com diversas limitações., Primeiramente, foi um estudo retrospectivo. Além disso, não foi feita distinção da técnica utilizada ou método de inserção do parafuso pedicular. Pontos como o centro da faceta, e o centro da cabeça dos parafusos/barra podem gerar dúvidas. Por fim, devemos mencionar o uso de u.a. Por mais que possam auxiliar na padronização de medidas, elas não são unidades validadas, podendo ter sua generalização restringida.

Apesar destas limitações, o presente estudo pode apontar direções para o futuro. Pois esses valores de referência $(\Delta$ ângulo e $\Delta$ distância) podem ser adicionados ao uso de imagens intraoperatórias ou de novas tecnologias como robótica ou navegação cirúrgica para aumentar a segurança na colocação de parafusos pediculares.

\section{Conclusão}

Esse trabalho demonstrou que a utilização de medidas que relacionam as facetas e os parafusos pediculares ( $\Delta$ ângulo e $\Delta$ distância) são efetivas para indicar e, quem sabe, no futuro, diminuir o risco de violação facetária. No entanto, outros estudos com diferentes populações utilizando as medidas propostas são necessários para verificar a aplicabilidade real da utilização dessas medidas.

Conflitos de interesse

Os autores declaram não haver conflitos de interesse.

\section{Referências}

1 Waldrop R, Cheng J, Devin C, McGirt M, Fehlings M, Berven S. The Burden of Spinal Disorders in the Elderly. Neurosurgery 2015;77 (Suppl 4):S46-S50

2 Buser Z, Ortega B, D'Oro A, et al. Spine Degenerative Conditions and Their Treatments: National Trends in the United States of America. Global Spine J 2018;8(01):57-67

3 Narain AS, Hijji FY, Markowitz JS, Kudaravalli KT, Yom KH, Singh K. Minimally invasive techniques for lumbar decompressions and fusions. Curr Rev Musculoskelet Med 2017;10(04):559-566

4 Tamburrelli FC, Meluzio MC, Burrofato A, Perna A, Proietti L. Minimally invasive surgery procedure in isthmic spondylolisthesis. Eur Spine J 2018;27(Suppl 2):237-243

5 Mobbs RJ, Phan K, Malham G, Seex K, Rao PJ. Lumbar interbody fusion: techniques, indications and comparison of interbody fusion options including PLIF, TLIF, MI-TLIF, OLIF/ATP, LLIF and ALIF. J Spine Surg 2015;1(01):2-18

6 Teles AR, Paci M, Gutman G, et al. Anatomical and technical factors associated with superior facet joint violation in lumbar fusion. J Neurosurg Spine 2018;28(02):173-180

7 Kim H-J, Chun HJ, Kang KT, et al. The biomechanical effect of pedicle screws' insertion angle and position on the superior adjacent segment in 1 segment lumbar fusion. Spine (Phila Pa 1976) 2012;37(19):1637-1644 
8 Matsukawa K, Kato T, Yato Y, et al. Incidence and Risk Factors of Adjacent Cranial Facet Joint Violation Following Pedicle Screw Insertion Using Cortical Bone Trajectory Technique. Spine (Phila Pa 1976) 2016;41(14):E851-E856

9 Magerl FP. Stabilization of the lower thoracic and lumbar spine with external skeletal fixation. Clin Orthop Relat Res 1984;(189): 125-141

10 Roy-Camille R, Saillant G, Mazel C. Internal fixation of the lumbar spine with pedicle screw plating. Clin Orthop Relat Res 1986; (203):7-17

11 Weinstein JN, Spratt KF, Spengler D, Brick C, Reid S. Spinal pedicle fixation: reliability and validity of roentgenogram-based assessment and surgical factors on successful screw placement. Spine (Phila Pa 1976) 1988;13(09):1012-1018

12 Zhang D, Gao X, Jiang J, Shen Y, Ding W, Cui H. Safe placement of pedicle screw in lumbar spine with minimum three year follow-up: a case series and technical note. Int Orthop 2018;42(03):567-573

13 Jones-Quaidoo SM, Djurasovic M, Owens RK 2nd, Carreon LY. Superior articulating facet violation: percutaneous versus open techniques. J Neurosurg Spine 2013;18(06):593-597

14 Knox JB, Dai JM III, Orchowski JR. Superior segment facet joint violation and cortical violation after minimally invasive pedicle screw placement. Spine J 2011;11(03):213-217

15 Archavlis E, Amr N, Kantelhardt SR, Giese A. Rates of Upper Facet Joint Violation in Minimally Invasive Percutaneous and Open Instrumentation: A Comparative Cohort Study of Different Insertion Techniques. J Neurol Surg A Cent Eur Neurosurg 2018;79(01): $1-8$
16 Wang L, Wang Y, Yu B, Li Z, Li Y. Comparison of cranial facet joint violation rate between percutaneous and open pedicle screw placement: a systematic review and meta-analysis. Medicine (Baltimore) 2015;94(05):e504

17 Xu Z, Tao Y, Li H, Chen G, Li F, Chen Q. Facet angle and its importance on joint violation in percutaneous pedicle screw fixation in lumbar vertebrae: A retrospective study. Medicine (Baltimore) 2018;97(22):e10943

18 Tannous O, Jazini E, Weir TB, et al. Facet Joint Violation During Percutaneous Pedicle Screw Placement: A Comparison of Two Techniques. Spine (Phila Pa 1976) 2017;42(15):1189-1194

19 Wang H, Ma L, Yang D, et al. Incidence and risk factors of adjacent segment disease following posterior decompression and instrumented fusion for degenerative lumbar disorders. Medicine (Baltimore) 2017;96(05):e6032

20 Babu R, Park JG, Mehta AI, et al. Comparison of superior-level facet joint violations during open and percutaneous pedicle screw placement. Neurosurgery 2012;71(05):962-970

21 Gao S, Lv Z, Fang H. Robot-assisted and conventional freehand pedicle screw placement: a systematic review and meta-analysis of randomized controlled trials. Eur Spine J 2018;27(04):921-930

22 Celik SE, Celik S, Kara A, Ince I, Göksu K. Lumbar facet joint angle and its importance on joint violation in lumbar microdiscectomy. Neurosurgery 2008;62(01):168-172

23 Jia L, Yu Y, Khan K, et al. Superior Facet Joint Violations during Single Level Minimally Invasive Transforaminal Lumbar Interbody Fusion: A Preliminary Retrospective Clinical Study [Internet]. Biomed Res Int 2018;2018:6152769 